\title{
2004 Population and Public Health (PPH) Student Award Program
}
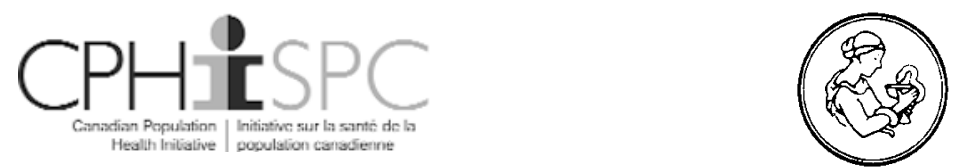

\section{Le Programme de bourses d'études en santé publique et de la population (SPP) de 2004}

\section{Preamble}

$\mathrm{T}$ The 2004 inaugural Population and Public Health (PPH) Student Award Program, jointly sponsored by the Canadian Institutes of Health Research-Institute of Population and Public Health (CIHR-IPPH), the Canadian Public Health Association (CPHA) and the Canadian Institute for Health Information's Canadian Population Health Initiative (CIHI$\mathrm{CPHI}$, was developed to acknowledge excellence in young PPH researchers, policy-makers and practitioners and awards were available to Masters and $\mathrm{PhD}$ students enrolled in Canadian academic institutions. The awards were presented at CPHA's 95th Annual Conference, held in St. John's, Newfoundland and Labrador, June 13-16, 2004.

The award winners and their research projects, representing diverse topics in PPH research, are: Sarah Viehbeck (MA candidate, Department of Health Studies and Gerontology, University of Waterloo)*, "Tobacco use and public health"; Jena Webb (MA candidate, Université de Québec à Montréal), "The use of the ecosystem approach to population health: The case of mercury contamination in aquatic environments and riparian populations, Napo River Valley, Ecuador"; Sharon Yanicki (MSc candidate, School of Health Studies, University of Lethbridge) ${ }^{\dagger}$, "Social support and family strengths: How do low-income single mothers view support received from a home visitation program?"; Alice Chen (PhD candidate, Department of Health Care and Epidemiology, University of British Columbia), "Utilization of mental health services by Chinese immigrants in British Columbia."

This inaugural program was well received by all who participated: awardees expressed appreciation for the support and encouragement, and felt that the award would positively impact on their future research careers; the sponsors and reviewers felt that the awards program was a positive method by which to recognize and encourage the next generation of PPH researchers, policy-makers and practitioners. Plans are underway to offer the program again. Successful applicants will be announced at CPHA's 96th annual conference, to be held in Ottawa (September 18-20, 2005).

\section{Préambule}

T e Programme inaugural de bourses d'études en santé publique et de la population (SPP) de 2004, parrainé conJjointement par l'Institut de la santé publique et des populations (ISPP) des Instituts de recherche en santé du Canada (IRSC), par l'Association canadienne de santé publique (ACSP) et par l'Initiative pour la santé de la population canadienne (ISPC) de l'Institut canadien d'information sur la santé (ICIS), vise à reconnaître l'excellence chez les jeunes chercheurs, les responsables des politiques et les praticiens en SPP. Il était ouvert aux étudiants à la maîtrise et au doctorat fréquentant des établissements d'enseignement au Canada. Les bourses ont été présentées au cours de la 95 Conférence annuelle de l'ACSP, tenue à St. John's (TerreNeuve-et-Labrador) du 13 au 16 juin 2004.

Les boursières et leurs projets de recherche, qui représentent bien les divers thèmes de la recherche en SPP, étaient : Sarah Viehbeck (candidate à la maîtrise, Département des études en santé et de gérontologie, Université de Waterloo)*, "Examen du lien entre le poids de la réglementation antitabac des municipalités et la probabilité d'être un ancien fumeur "; Jena Webb (candidate à la maîtrise, Université du Québec à Montréal), "L'utilisation de l'approche écosystémique en santé des populations : la contamination par le mercure des environnements aquatiques et des populations riveraines, vallée du Napo, Équateur "; Sharon Yanicki (candidate à la maîtrise en sciences, École d'études en santé, Université de Lethbridge) $)^{\dagger}$, "Appui social et forces familiales : ce que les mères célibataires à faible revenu pensent de l'appui reçu d'un programme de visites à domicile "; Alice Chen (candidate au doctorat, Département des soins de santé et d'épidémiologie, Université de la Colombie-Britannique), "Utilisation des services de santé mentale par les immigrants chinois en Colombie-Britannique ».

Ce programme inaugural a été bien accueilli par tous les participants : les boursières ont exprimé leur reconnaissance pour l'appui et l'encouragement reçus et considèrent que la bourse aura des répercussions positives sur leur carrière en recherche. Les commanditaires et les examinateurs sont d'avis que le programme de bourses constitue un moyen positif de reconnaître et d'encourager la prochaine génération de chercheurs, de décideurs et de praticiens en SPP. On planifie déjà la deuxième édition du programme. Les noms des prochains boursiers et boursières seront annoncés au cours de la $96^{\mathrm{e}}$ Conférence annuelle de l'ACSP, qui aura lieu l'an prochain à Ottawa (du 18 au 20 septembre 2005).

Sarah Viehbeck a défendu sa thèse et est actuellement inscrite à un programme de doctorat à l'Université de Waterloo.

† Sharon Yanicki a terminé sa maîtrise en sciences à l'Université de l'Alberta et travaille comme consultante en promotion de la santé à Lethbridge (Alberta). 